CORRESPONDENCE

\section{Sudden cardiac death is associated both with epilepsy and with use of antiepileptic drugs}

To the Editor, We read with interest the recent paper by Bardai et al, ${ }^{1}$ which reports that epilepsy and antiepileptic drugs (AEDs) were independently associated with sudden cardiac death (SCD). We are unconvinced that such a clear distinction between disease and drug effects can be made in this study as all people with epilepsy were by definition taking AEDs. We believe this is why SCD risk in those with epilepsy (table 2) and in AED users with epilepsy (table 3) was the same: OR 2.8 (95\% CI 1.4 to 5.3). It is unclear whether these numbers reflect disease effects, drug effects or both.

It is reported that people with epilepsy are at increased risk of SCD, yet in this population there is a major diagnostic alternative: sudden unexpected death in epilepsy, an autopsy-negative mostly seizure-related type of sudden death with cardiac and noncardiac causes. ${ }^{2}$ We do not believe that sudden death in people with epilepsy can be assumed to be of cardiac origin (vs, for example neurogenic respiratory depression) without documentation of a cardiac mechanism of death (eg, by ECG).

Carbamazepine and gabapentin were the only individual AEDs associated with increased SCD risk. The authors attribute this to the supposed sodium channel blocking properties of these drugs, but we believe that indication bias cannot be excluded. Carbamazepine is the drug of choice in people with focal seizures and stroke is a leading cause of this type of epilepsy, particularly in the elderly. ${ }^{3}$ An important indication for gabapentin is diabetic chronic neuropathic pain. ${ }^{4}$ It is possible that a worse cardiovascular status rather than sodium channel blocking properties explains the higher SCD risk in users of these AEDs.

Despite the use of a large database, many numbers are small, so the demonstrated significant effect for sodium channel blocking AEDs but not for nonsodium channel blocking AEDs may reflect limited sample size rather than differences in effect. There was a consistent trend; all reported AED ORs were greater than the null value.

This paper is important in that it confirms that epilepsy in the community is associated with an increased risk of sudden natural death. Future studies with additional documentation of death mechanisms, correction for indication bias and larger sample size are needed to explain the cause of this excess risk and clarify the role of AEDs.

Prisca R Bauer, Jan Novy, Mark R Keezer, Gail S Bell

NIHR University College London Hospitals Biomedical Research Centre, Clinical \& Experimental Epilepsy, UCL Institute of Neurology, London, UK

Correspondence to Prisca R Bauer, NIHR University College London Hospitals Biomedical Research Centre, UCL Institute of Neurology, Queen Square, London WC1N 3BG, UK; prisca.bauer.12@ucl.ac.uk

Contributors All authors were involved in writing the final manuscript.

Competing interests None.

Provenance and peer review Commissioned; internally peer reviewed.

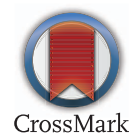

To cite Bauer PR, Novy J, Keezer MR, et al. Heart 2015;101:83.

Published Online First 25 September 2014

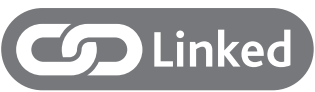

- http://dx.doi.org/10.1136/heartjnl-2014-305664

Heart 2015;101:83.

doi:10.1136/heartjnl-2014-306760

\section{REFERENCES}

1 Bardai A, Blom MT, van Noord C, et al. Sudden cardiac death is associated both with epilepsy and with use of antiepileptic drugs. Heart 2015;101:17-22.

2 Shorvon S, Tomson T. Sudden unexpected death in epilepsy. Lancet 2011;378:2028-38.

3 Ryvlin P, Montavont A, Nighoghossian N. Optimizing therapy of seizures in stroke patients. Neurology 2006;67(12 Suppl 4):S3-9.

4 Moore RA, Wiffen PJ, Derry S, et al. Gabapentin for chronic neuropathic pain and fibromyalgia in adults. Cochrane Database Syst Rev 2014;4:CD007938. 